\title{
A Hierarchical Flexible Coarsening Method to Combine BBAs in Probabilities
}

\author{
Yilin Dong, Xinde Li \\ School of Automation, Southeast University \\ Nanjing, Jiangsu, China 210096. \\ Email:xindeli@seu.edu.cn
}

\author{
Jean Dezert \\ The French Aerospace Lab \\ F-91761 Palaiseau, France. \\ Email:jean.dezert@onera.fr
}

\begin{abstract}
In many applications involving epistemic uncertainties usually modeled by belief functions, it is often necessary to approximate general (non-Bayesian) basic belief assignments (BBAs) to subjective probabilities (called Bayesian BBAs). This necessity occurs if one needs to embed the fusion result in a system based on the probabilistic framework and Bayesian inference (e.g. tracking systems), or if one wants to use classical decision theory to make a decision. There exists already several methods (probabilistic transforms) to approximate any general BBA to a Bayesian BBA. From a fusion standpoint, two approaches are usually adopted: 1) one can approximate at first each BBA in subjective probabilities and use Bayes fusion rule to get the final Bayesian BBA, or 2) one can fuse all the BBAs with a fusion rule, typically Dempster-Shafer's, or PCR6 rules (which is very costly in computations), and convert the combined BBA in a subjective probability measure. The former method is the simplest method but it generates a high loss of information included in original BBAs, whereas the latter is intractable for high dimension problems. This paper presents a new method to achieve this task based on hierarchical decomposition (coarsening) of the frame of discernment, which can be seen as an intermediary approach between the two aforementioned methods. After the presentation of this new method, we show through simulations how its performs with respect to other methods.
\end{abstract}

Keywords: Information fusion, belief functions, DST, DSmT, PCR6 rule, coarsening.

\section{INTRODUCTION}

The theory of belief functions, known as Dempster-Shafer Theory (DST) has been developed by Shafer [1] in 1976 from Dempster's works [2]. Belief functions allow to model epistemic uncertainty and they have been already used in many applications since the 1990's [3], mainly those related to expert systems, decision-making support and information fusion. To palliate some limitations of DST, Dezert and Smarandache have proposed an extended mathematical framework of belief functions with new efficient quantitative and qualitative rules of combinations, which is called DSmT (Dezert and Smarandache Theory) in the literature [4], [5] with applications listed in [6]. One of the major drawbacks of DST and DSmT is their high computational complexities, as soon as the fusion space (i.e. frame of discernment - FoD) and the number of sources to combine are large ${ }^{1}$.

\footnotetext{
${ }^{1} \mathrm{DSmT}$ is more complex than DST, and the Proportional Conflict Redistribution rule \#6 (PCR6 rule) becomes computationally intractable in the worst case as soon as the cardinality of the Frame of Discernment (FoD) is greater than six
}

To reduce the computational cost of operations with belief functions when the number of focal elements is very large, several approaches have been proposed by different authors. Basically, the existing approaches rely either on efficient implementations of computations as proposed for instance in [7], [8], or on approximation techniques of original Basic Belief Assignment (BBA) to combine [9]-[12], or both. In many applications involving epistemic uncertainties usually modeled by belief functions, it is often necessary to approximate general (non-Bayesian) basic belief assignments (BBAs) to subjective probabilities (called Bayesian BBAs). This necessity occurs if one needs to embed the fusion result in a system based on the probabilistic framework and Bayesian inference (e.g. tracking systems), or if one wants to use classical decision theory to make a decision. From a fusion standpoint, two approaches are usually adopted: 1) one can approximate at first each BBA in subjective probabilities and use Bayes fusion rule to get the final Bayesian BBA, or 2) one can fuse all the BBAs with a fusion rule, typically Dempster-Shafer's, or PCR6 rules (which is very costly in computations), and convert the combined BBA in a subjective probability measure. The former method is the simplest method but it generates a high loss of information included in original BBAs, whereas the latter direct method is intractable for high dimension problems. This paper presents a new method to achieve this task based on hierarchical decomposition (coarsening) of the frame of discernment, which can be seen as an intermediary approach between the two aforementioned methods.

This paper presents a new approach to fuse BBAs into a Bayesian BBA in order to reduce computational burden and keep the fusion tractable even for large dimension problems. This method is based on a hierarchical decomposition (coarsening) framework which allows to keep as much as possible information of original BBAs in preserving lower complexity. The main contributions of this paper are:

1) the presentation of the FoD bintree decomposition on which will be done the BBAs approximations;

2) the presentation of the fusion of approximate BBAs from bintree representation.

This hierarchical structure allows to encompass bintree decomposition and BBAs approximations on it to obtain the final approximate fusionned Bayesian BBA. 
This paper is organized as follows. In section II, we recall some basics of DST and DSmT that are relevant to the new method presented in this paper. More details with examples can easily be found in [1], [5]. We will also briefly recall our preliminary works about hierarchical coarsening of FoD. Section III presents the novel hierarchical flexible (adaptive) coarsening method which can be regarded as the extension of our previous works. Two simple examples are given in section IV to illustrate the detailed calculation steps. Simulation experiments are presented in section $\mathrm{V}$ to show the rationality of this new approach. Finally, Sect.VI concludes the paper with future works perspectives.

\section{MathematicAl BACKGROUND}

This section provides a brief reminder of basics of DST and DSmT, and of original hierarchical coarsening method which are necessary for the presentation and the understanding of the more general flexible coarsening approximate method of section III.

\section{A. Basics of DST and DSmT}

In DST framework, the frame of discernment ${ }^{2} \Theta \triangleq$ $\left\{\theta_{1}, \ldots, \theta_{n}\right\}(n \geq 2)$ is a set of exhaustive and exclusive elements (hypotheses) which represent the possible solutions of the problem under consideration and thus Shafer's model assumes $\theta_{i} \cap \theta_{j}=\emptyset$ for $i \neq j$ in $\{1, \ldots, n\}$. A basic belief assignment (BBA) $m(\cdot)$ is defined by the mapping: $2^{\Theta} \mapsto[0,1]$, verifying $m(\emptyset)=0$ and $\sum_{A \in 2^{\Theta}} m(A)=1$. In DSmT, one can abandon Shafer's model (if Shafer's model doesn't fit with the problem) and refute the principle of the third excluded middle ${ }^{3}$. Instead of defining the BBAs on the power set $2^{\Theta} \triangleq(\Theta, \cup)$ of the FoD, the BBAs are defined on the so-called hyper-power set (or Dedekind's lattice) denoted $D^{\Theta} \triangleq(\Theta, \cup, \cap)$ whose cardinalities follows Dedekind's numbers sequence, see [5], Vol.1 for details and examples. A (generalized) BBA, called a mass function, $m(\cdot)$ is defined by the mapping: $D^{\Theta} \mapsto[0,1]$, verifying $m(\emptyset)=0$ and $\sum_{A \in D^{\Theta}} m(A)=1$. DSmT framework encompasses DST framework because $2^{\Theta} \subset D^{\Theta}$. In DSmT we can take into account also a set of integrity constraints on the FoD (if known), by specifying all the pairs of elements which are really disjoint. Stated otherwise, Shafer's model is a specific DSm model where all elements are known to be disjoint. $A \in D^{\Theta}$ is called a focal element of $m($.$) if m(A)>0$. A BBA is called a Bayesian BBA if all of its focal elements are singletons and Shafer's model is assumed, otherwise it is called nonBayesian [1]. A full ignorance source is represented by the vacuous BBA $m_{v}(\Theta)=1$. The belief (or credibility) and plausibility functions are respectively defined by $\operatorname{Bel}(X) \triangleq$ $\sum_{Y \in D^{\Theta} \mid Y \subseteq X} m(Y)$ and $P l(X) \triangleq \sum_{Y \in D^{\Theta} \mid Y \cap X \neq \emptyset} m(Y)$. $B I(X) \triangleq[\operatorname{Bel}(X), P l(X)]$ is called the belief interval of $X$. Its length $U(X) \triangleq P l(X)-\operatorname{Bel}(X)$ measures the degree of uncertainty of $X$.

\footnotetext{
${ }^{2}$ We use the symbol $\triangleq$ to mean equals by definition.

${ }^{3}$ The third excluded middle principle assumes the existence of the complement for any elements/propositions belonging to the power set $2^{\Theta}$.
}

In 1976, Shafer did propose Dempster's rule ${ }^{4}$ to combine BBAs in DST framework. DS rule is defined by $m_{D S}(\emptyset)=0$ and $\forall A \in 2^{\Theta} \backslash\{\emptyset\}$,

$$
m_{D S}(A)=\frac{\sum_{B, C \in 2^{\Theta} \mid B \cap C=A} m_{1}(B) m_{2}(C)}{1-\sum_{B, C \in 2^{\Theta} \mid B \cap C=\emptyset} m_{1}(B) m_{2}(C)}
$$

DS rule formula is commutative and associative and can be easily extended to the fusion of $S>2$ BBAs. Unfortunately, DS rule has been highly disputed during the last decades by many authors because of its counter-intuitive behavior in high or even low conflict situations, and that is why many rules of combination have been proposed in the literature to combine BBAs [13]. To palliate DS rule drawbacks, the very interesting PCR6 (Proportional Conflict redistribution rule \#6) has been proposed in DSmT and it is usually adopted ${ }^{5}$ in recent applications of DSmT. The fusion of two BBAs $m_{1}($. and $m_{2}($.$) by the PCR6 rule is obtained by m_{P C R 6}(\emptyset)=0$ and $\forall A \in D^{\Theta} \backslash\{\emptyset\}$

$$
\begin{aligned}
& m_{P C R 6}(A)=m_{12}(A)+ \\
& \sum_{B \in D^{\Theta} \backslash\{A\} \mid A \cap B=\emptyset}\left[\frac{m_{1}(A)^{2} m_{2}(B)}{m_{1}(A)+m_{2}(B)}+\frac{m_{2}(A)^{2} m_{1}(B)}{m_{2}(A)+m_{1}(B)}\right]
\end{aligned}
$$

where $m_{12}(A)=\sum_{B, C \in D^{\Theta} \mid B \cap C=A} m_{1}(B) m_{2}(C)$ is the conjunctive operator, and each element $A$ and $B$ are expressed in their disjunctive normal form. If the denominator involved in the fraction is zero, then this fraction is discarded. The general PCR6 formula for combining more than two BBAs altogether is given in [5], Vol. 3. We adopt the generic notation $m_{12}^{P C R 6}()=.\operatorname{PCR6}\left(m_{1}(),. m_{2}().\right)$ to denote the fusion of $m_{1}($.$) and m_{2}($.$) by PCR6 rule. PCR6 is not associative$ and PCR6 rule can also be applied in DST framework (with Shafer's model of FoD) by replacing $D^{\Theta}$ by $2^{\Theta}$ in Eq. (2).

\section{B. Hierarchical coarsening for fusion of Bayesian BBAs}

Here, we briefly recall the principle of hierarchical coarsening of FoD to reduce the computational complexity of PCR6 combination of original Bayesian BBAs. The fusion of original non-Bayesian BBAs will be presented in the next section.

This principle was called rigid grouping in our previous works [17]-[19]. The goal of this coarsening is to replace the original (refined) Frame of Discernment (FoD) $\Theta$ by a set of coarsened ones to make the computation of PCR6 rule tractable. Because we consider here only Bayesian BBA to combine, their focal elements are only singletons of the FoD $\Theta \triangleq\left\{\theta_{1}, \ldots, \theta_{n}\right\}$, with $n \geq 2$, and we assume Shafer's model of the FoD $\Theta$.

A coarsening of the FoD $\Theta$ means to replace it with another FoD less specific of smaller dimension $\Omega=\left\{\omega_{1}, \ldots, \omega_{k}\right\}$ with $k<n$ from the elements of $\Theta$. This can be done in many ways depending the problem under consideration. Generally, the elements of $\Omega$ are singletons of $\Theta$, and disjunctions of

\footnotetext{
${ }^{4}$ We use DS index to refer to Dempster-Shafer's rule (DS rule) because Shafer did really promote Dempster's rule in in his milestone book [1].

${ }^{5}$ PCR6 rule coincides with PCR5 when combining only two BBAs [5].
} 
elements of $\Theta$. For example, if $\Theta=\left\{\theta_{1}, \theta_{2}, \theta_{3}, \theta_{4}\right\}$, then the possible coarsened frames built from $\Theta$ could be, for instance, $\Omega=\left\{\omega_{1}=\theta_{1}, \omega_{2}=\theta_{2}, \omega_{3}=\theta_{3} \cup \theta_{4}\right\}$, or $\Omega=\left\{w_{1}=\right.$ $\left.\theta_{1} \cup \theta_{2}, \omega_{2}=\theta_{3} \cup \theta_{4}\right\}$, etc. When dealing with Bayesian BBAs, the projection ${ }^{6} m^{\Omega}($.$) of the original BBA m^{\Theta}($.$) is simply$ obtained by taking

$$
m^{\Omega}\left(\omega_{i}\right)=\sum_{\theta_{j} \subseteq \omega_{i}} m^{\Theta}\left(\theta_{j}\right)
$$

The hierarchical coarsening process (or rigid grouping) is a simple dichotomous approach of coarsening obtained as follows:

- If $n=|\Theta|$ is an even number:

The disjunction of the $n / 2$ first elements $\theta_{1}$ to $\theta_{\frac{n}{2}}$ of $\Theta$ define the element $\omega_{1}$ of $\Omega$, and the last $n / 2$ elements $\theta_{\frac{n}{2}+1}$ to $\theta_{n}$ of $\Theta$ define the element $\omega_{2}$ of $\Omega$, that is

$$
\Omega \triangleq\left\{\omega_{1}=\theta_{1} \cup \ldots \cup \theta_{\frac{n}{2}}, \omega_{2}=\theta_{\frac{n}{2}+1} \cup \ldots \cup \theta_{n}\right\}
$$

and based on (3), one has

$$
\begin{aligned}
m^{\Omega}\left(\omega_{1}\right) & =\sum_{j=1, \ldots, \frac{n}{2}} m^{\Theta}\left(\theta_{j}\right) \\
m^{\Omega}\left(\omega_{2}\right) & =\sum_{j=\frac{n}{2}+1, \ldots, n} m^{\Theta}\left(\theta_{j}\right)
\end{aligned}
$$

For example, if $\Theta=\left\{\theta_{1}, \theta_{2}, \theta_{3}, \theta_{4}\right\}$, and one considers the Bayesian BBA $m^{\Theta}\left(\theta_{1}\right)=0.1, m^{\Theta}\left(\theta_{2}\right)=0.2$, $m^{\Theta}\left(\theta_{3}\right)=0.3$ and $m^{\Theta}\left(\theta_{4}\right)=0.4$, then $\Omega=\left\{\omega_{1}=\right.$ $\left.\theta_{1} \cup \theta_{2}, \omega_{2}=\theta_{3} \cup \theta_{4}\right\}$ and $m^{\Omega}\left(\omega_{1}\right)=0.1+0.2=0.3$ and $m^{\Omega}\left(\omega_{2}\right)=0.3+0.4=0.7$.

- If $n=|\Theta|$ is an odd number:

In this case, the element $\omega_{1}$ of the coarsened frame $\Omega$ is the disjunction of the $[n / 2+1]^{7}$ first elements of $\Theta$, and the element $\omega_{2}$ is the disjunction of other elements of $\Theta$. That is

$\Omega \triangleq\left\{\omega_{1}=\theta_{1} \cup \ldots \cup \theta_{\left[\frac{n}{2}+1\right]}, \omega_{2}=\theta_{\left[\frac{n}{2}+1\right]+1} \cup \ldots \cup \theta_{n}\right\}$

and based on (3), one has

$$
\begin{gathered}
m^{\Omega}\left(\omega_{1}\right)=\sum_{j=1, \ldots,\left[\frac{n}{2}+1\right]} m^{\Theta}\left(\theta_{j}\right) \\
m^{\Omega}\left(\omega_{2}\right)=\sum_{j=\left[\frac{n}{2}+1\right]+1, \ldots, n} m^{\Theta}\left(\theta_{j}\right)
\end{gathered}
$$

For example, if $\Theta=\left\{\theta_{1}, \theta_{2}, \theta_{3}, \theta_{4}, \theta_{5}\right\}$, and one considers the Bayesian BBA $m^{\Theta}\left(\theta_{1}\right)=0.1, m^{\Theta}\left(\theta_{2}\right)=0.2$, $m^{\Theta}\left(\theta_{3}\right)=0.3, m^{\Theta}\left(\theta_{4}\right)=0.3$ and $m^{\Theta}\left(\theta_{5}\right)=0.1$, then $\Omega=\left\{\omega_{1}=\theta_{1} \cup \theta_{2} \cup \theta_{3}, \omega_{2}=\theta_{4} \cup \theta_{5}\right\}$ and $m^{\Omega}\left(\omega_{1}\right)=$ $0.1+0.2+0.3=0.6$ and $m^{\Omega}\left(\omega_{2}\right)=0.3+0.1=0.4$.

Of course, the same coarsening applies to all original BBAs $m_{s}^{\Theta}(),. s=1, \ldots S$ of the $S>1$ sources of evidence to work with less specific BBAs $m_{s}^{\Omega}(),. s=1, \ldots S$. The less specific

${ }^{6}$ For clarity and convenience, we put explicitly as upper index the FoD for which the belief mass refers.

${ }^{7}$ The notation $[x]$ means the integer part of $x$.
BBAs (called coarsened BBAs by abuse of language) can then be combined with PCR6 rule of combination according to formula (2). This dichotomous coarsening method is repeated iteratively $l$ times as schematically represented by a bintree ${ }^{8}$. The last step of this hierarchical process is to calculate the combined (Bayesian) BBA of all focal elements according to the connection weights of the bintree structure, where the number of iterations (or layers) $l$ of the tree depends on the cardinality $|\Theta|$ of the original FoD $\Theta$. Specifically, the assignment of each focal element is updated according to the connection weights of link paths from root to terminal nodes. This principle is illustrated in details in the following example.

Example 1: Let's consider $\Theta=\left\{\theta_{1}, \theta_{2}, \theta_{3}, \theta_{4}, \theta_{5}\right\}$, and the following three Bayesian BBAs

\begin{tabular}{c|c|c|c} 
Focal elem. & $m_{1}^{\Theta}()$. & $m_{2}^{\Theta}()$. & $m_{3}^{\Theta}()$. \\
\hline$\theta_{1}$ & 0.1 & 0.4 & 0 \\
$\theta_{2}$ & 0.2 & 0 & 0.1 \\
$\theta_{3}$ & 0.3 & 0.1 & 0.5 \\
$\theta_{4}$ & 0.3 & 0.1 & 0.4 \\
$\theta_{5}$ & 0.1 & 0.4 & 0
\end{tabular}

The hierarchical coarsening and fusion of BBAs is obtained from the following steps:

Step 1: We define the bintree structure based on iterative half split of FoD as shown in Fig. 1.

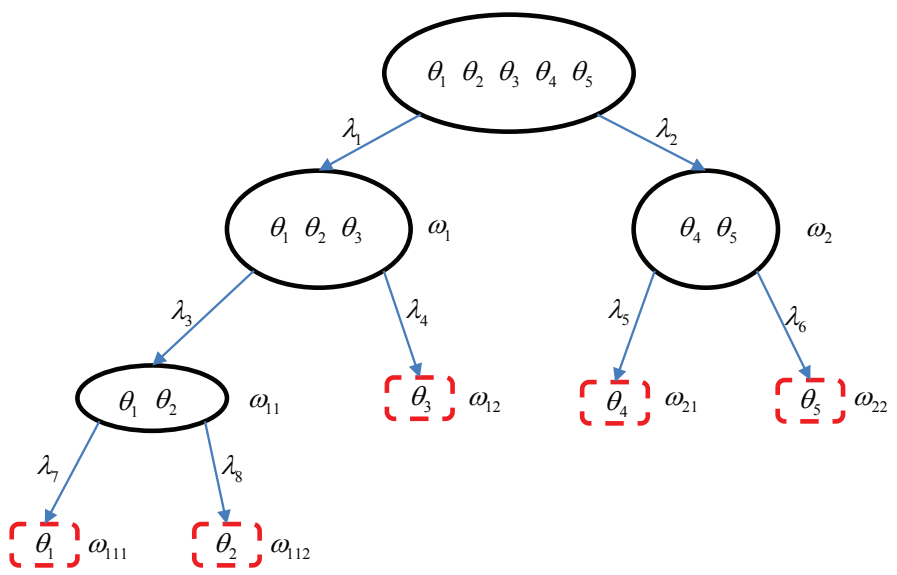

Figure 1: Fusion of Bayesian BBAs using bintree coarsening for Example 1.

The connecting weights are denoted as $\lambda_{1}, \ldots, \lambda_{8}$. The elements of the frames $\Omega_{l}$ are defined as follows:

- At layer $l=1: \Omega_{1}=\left\{\omega_{1} \triangleq \theta_{1} \cup \theta_{2} \cup \theta_{3}, \omega_{2} \triangleq \theta_{4} \cup \theta_{5}\right\}$

- At layer $l=2$ :

$$
\Omega_{2}=\left\{\omega_{11} \triangleq \theta_{1} \cup \theta_{2}, \omega_{12} \triangleq \theta_{3}, \omega_{21} \triangleq \theta_{4}, \omega_{22}=\theta_{5}\right\}
$$

- At layer $l=3: \Omega_{3}=\left\{\omega_{111} \triangleq \theta_{1}, \omega_{112} \triangleq \theta_{2}\right\}$

\footnotetext{
${ }^{8}$ Here we consider bintree only for simplicity, which means that the coarsened frame $\Omega$ consists of two elements only. Of course a similar method can be used with tri-tree, quad-tree, etc.
} 
Step 2: The BBAs of elements of the (sub-)frames $\Omega_{l}$ are obtained as follows:

- At layer $l=1$, we use (6)-(7) because $|\Theta|=5$ is an odd number. Therefore, we get

\begin{tabular}{c|c|c|c} 
Focal elem. & $m_{1}^{\Omega_{1}}()$. & $m_{2}^{\Omega_{1}}()$. & $m_{3}^{\Omega_{1}}()$. \\
\hline$\omega_{1} \triangleq \theta_{1} \cup \theta_{2} \cup \theta_{3}$ & 0.6 & 0.5 & 0.6 \\
$\omega_{2} \triangleq \theta_{4} \cup \theta_{5}$ & 0.4 & 0.5 & 0.4
\end{tabular}

- At layer $l=2$ : We work with the two subframes $\Omega_{21} \triangleq$ $\left\{\omega_{11}, \omega_{12}\right\}$ and $\Omega_{22} \triangleq\left\{\omega_{21}, \omega_{22}\right\}$ of $\Omega_{2}$ with the BBAs:

\begin{tabular}{c|c|c|c} 
Focal elem. & $m_{1}^{\Omega_{21}}()$. & $m_{2}^{\Omega_{21}}()$. & $m_{3}^{\Omega_{21}}()$. \\
\hline$\omega_{11} \triangleq \theta_{1} \cup \theta_{2}$ & $\frac{1}{2}$ & $\frac{4}{5}$ & $\frac{1}{6}$ \\
$\omega_{12} \triangleq \theta_{3}$ & $\frac{1}{2}$ & $\frac{1}{5}$ & $\frac{5}{6}$ \\
Focal elem. & $m_{1}^{\Omega_{22}}()$. & $m_{2}^{\Omega_{22}}()$. & $m_{3}^{\Omega_{22}(.)}$ \\
\hline$\omega_{21} \triangleq \theta_{4}$ & $\frac{3}{4}$ & $\frac{1}{5}$ & 1 \\
$\omega_{22} \triangleq \theta_{5}$ & $\frac{1}{4}$ & $\frac{4}{5}$ & 0
\end{tabular}

These mass values are obtained by the proportional redistribution of the mass of each focal element with respect to the mass of its parent focal element in the bin tree. For example, the value $m_{2}^{\Omega_{21}}\left(\omega_{11}\right)=4 / 5$ is derived by taking

$m_{2}^{\Omega_{21}}\left(\omega_{11}\right)=\frac{m_{2}^{\Theta}\left(\theta_{1}\right)+m_{2}^{\Theta}\left(\theta_{2}\right)}{m_{2}^{\Theta}\left(\theta_{1}\right)+m_{2}^{\Theta}\left(\theta_{2}\right)+m_{2}^{\Theta}\left(\theta_{3}\right)}=\frac{0.4}{0.5}=\frac{4}{5}$

Other mass values are computed similarly using this proportional redistribution method.

- At layer $l=3$ : We use again the proportional redistribution method which gives us

\begin{tabular}{c|c|c|c} 
Focal elem. & $m_{1}^{\Omega_{3}}()$. & $m_{2}^{\Omega_{3}}()$. & $m_{3}^{\Omega_{3}}()$. \\
\hline$\omega_{111} \triangleq \theta_{1}$ & $\frac{1}{3}$ & 1 & 0 \\
$\omega_{112} \triangleq \theta_{2}$ & $\frac{2}{3}$ & 0 & 1
\end{tabular}

Step 3: The connection weights $\lambda_{i}$ are computed from the assignments of coarsening elements. In each layer $l$, we fuse sequentially ${ }^{9}$ the three BBAs using PCR6 formula (2). More precisely, we compute at first $m_{12}^{P C R 6, \Omega_{l}}()=.\operatorname{PCR} 6\left(m_{1}^{\Omega_{l}}(),. m_{2}^{\Omega_{l}}().\right)$ and then $m_{(12) 3}^{P C R 6, \Omega_{l}}()=.\operatorname{PCR} 6\left(m_{12}^{P C R 6, \Omega_{l}}(),. m_{3}^{\Omega_{l}}().\right)$. Hence, we obtain the following connecting weights in the bintree:

- At layer $l=1$ :

$$
\begin{aligned}
& \lambda_{1}=m_{(12) 3}^{P C R, \Omega_{1}}\left(\omega_{1}\right)=0.6297 \\
& \lambda_{2}=m_{(12) 3}^{P C R 6, \Omega_{1}}\left(\omega_{2}\right)=0.3703
\end{aligned}
$$

- At layer $l=2$ :

$$
\begin{aligned}
& \lambda_{3}=m_{(12) 3}^{P C R 6, \Omega_{21}}\left(\omega_{11}\right)=0.4137 \\
& \lambda_{4}=m_{(12) 3}^{P C R 6, \Omega_{21}}\left(\omega_{12}\right)=0.5863 \\
& \lambda_{5}=m_{(12) 3}^{P C R 6, \Omega_{22}}\left(\omega_{21}\right)=0.8121 \\
& \lambda_{6}=m_{(12) 3}^{P C R 6, \Omega_{22}}\left(\omega_{22}\right)=0.1879
\end{aligned}
$$

\footnotetext{
${ }^{9}$ Because PCR6 fusion is not associative, we should apply the general PCR6 formula to get best results. Here we use sequential fusion to reduce the computational complexity even if the fusion result is approximate.
}

- At layer $l=3$ :

$$
\begin{aligned}
& \lambda_{7}=m_{(12) 3}^{P C R 6, \Omega_{3}}\left(\omega_{111}\right)=0.3103 \\
& \lambda_{8}=m_{(12) 3}^{P C R 6, \Omega_{3}}\left(\omega_{112}\right)=0.6897
\end{aligned}
$$

Step 4: The final assignment of belief mass to the elements of original $\mathrm{FoD} \Theta$ are calculated using the product of the connection weights of link paths from root (top) node to terminal nodes (leaves). We finally get the following resulting combined and normalized Bayesian BBA

$$
\begin{aligned}
& m^{\Theta}\left(\theta_{1}\right)=\lambda_{1} \cdot \lambda_{3} \cdot \lambda_{7}=0.6297 \cdot 0.4137 \cdot 0.3103=0.0808 \\
& m^{\Theta}\left(\theta_{2}\right)=\lambda_{1} \cdot \lambda_{3} \cdot \lambda_{8}=0.6297 \cdot 0.4137 \cdot 0.6897=0.1797 \\
& m^{\Theta}\left(\theta_{3}\right)=\lambda_{1} \cdot \lambda_{4}=0.6297 \cdot 0.5863=0.3692 \\
& m^{\Theta}\left(\theta_{4}\right)=\lambda_{2} \cdot \lambda_{5}=0.3703 \cdot 0.8121=0.3007 \\
& m^{\Theta}\left(\theta_{5}\right)=\lambda_{2} \cdot \lambda_{6}=0.3703 \cdot 0.1879=0.0696
\end{aligned}
$$

\section{NEW HIERARCHICAL FLEXIBLE COARSENING METHOD}

Contrary to the (rigid) hierarchical coarsening method presented in section II, in our new flexible coarsening approach the elements $\theta_{i}, i=1, \ldots, n$ in FoD $\Theta$ will not be half split to build coarsening focal elements $\omega_{j}, j=1, \ldots, k$ of the FoD $\Omega_{l}$. In the hierarchical flexible (adaptive) coarsening method, the elements $\theta_{i}$ chosen to belong to the same group are determined using the consensus information drawn from the BBAs provided by the sources. Specifically, the degrees of disagreement between the provided sources on decisions $\left(\theta_{1}, \theta_{2}, \cdots, \theta_{n}\right)$ are first calculated using the belief-interval based distance $d_{B I}$ [16], [20] to obtain disagreement vector. Then, the k-means algorithm is applied for clustering elements $\theta_{i}, i=1, \ldots, n$ based on the corresponding value in consensus vector. It is worth noting that values of disagreement reflect the preferences of independent sources of evidence for the same focal element. If they are small, it means that all sources have a consistent opinion and these elements should be clustered in the same group. Conversely, if disagreement values are large, it means that the sources have strong disagreement on these focal elements, and these focal elements need to be clustered in another group.

\section{A. Calculating the disagreement vector}

Let us consider several BBAs $m_{s}^{\Theta}(\cdot),(s=1, \ldots, S)$ defined on same FoD $\Theta$ of cardinality $|\Theta|=n$. The specific BBAs $m_{\theta_{i}}(),. i=1, \ldots, n$ entirely focused on $\theta_{i}$ are defined by $m_{\theta_{i}}\left(\theta_{i}\right)=1$, and for $X \neq \theta_{i} m_{\theta_{i}}(X)=0$. The disagreement of opinions of two sources about $\theta_{i}$ is defined as the $L_{1}$ distance between the $d_{B I}$ distances of the BBAs $m_{s}^{\Theta}(),. s=$ 1,2 to $m_{\theta_{i}}($.$) , which is expressed by$

$$
\left.D_{12}\left(\theta_{i}\right) \triangleq \mid d_{B I}\left(m_{1}^{\Theta}(\cdot), m_{\theta_{i}}(\cdot)\right)\right)-d_{B I}\left(m_{2}^{\Theta}(\cdot), m_{\theta_{i}}(\cdot)\right) \mid
$$

The disagreement of opinions of $S \geq 3$ sources about $\theta_{i}$, is defined as

$$
\begin{aligned}
D_{1-S}\left(\theta_{i}\right) \triangleq \frac{1}{2} \sum_{i=1}^{S} \sum_{j=1}^{S} \mid d_{B I}\left(m_{i}^{\Theta}(\cdot), m_{\theta_{i}}(.)\right) & \\
& -d_{B I}\left(m_{j}^{\Theta}(\cdot), m_{\theta_{i}}(.)\right) \mid
\end{aligned}
$$


where $d_{B I}$ distance is defined by ${ }^{10}$ [20]

$$
d_{B I}^{E}\left(m_{1}, m_{2}\right) \triangleq \sqrt{n_{c} \cdot \sum_{i=1}^{2^{n}-1}\left[d^{I}\left(B I_{1}\left(\theta_{i}\right), B I_{2}\left(\theta_{i}\right)\right)\right]^{2}}
$$

Here, $n_{c}=1 / 2^{n-1}$ is the normalization constant and $d^{I}([a, b],[c, d])$ is the Wasserstein's distance defined by $d^{I}([a, b],[c, d])=\sqrt{\left[\frac{a+b}{2}-\frac{c+d}{2}\right]^{2}+\frac{1}{3}\left[\frac{b-a}{2}-\frac{d-c}{2}\right]^{2}}$. And $B I\left(\theta_{i}\right)=\left[\operatorname{Bel}\left(\theta_{i}\right), \operatorname{Pl}\left(\theta_{i}\right)\right]$.

The disagreement vector $\mathbf{D}_{1-S}$ is defined by

$$
\mathbf{D}_{1-S} \triangleq\left[D_{1-S}\left(\theta_{1}\right), \ldots, D_{1-S}\left(\theta_{n}\right)\right]
$$

\section{B. Clustering focal elements}

Once $\mathbf{D}_{1-S}$ is derived, a clustering algorithm is used to coarsen focal elements according to their corresponding values in $\mathbf{D}_{1-S}$. In this paper, we have used the k-means algorithm ${ }^{11}$ to cluster focal elements. For each source $s=1, \ldots, S$, the mass assignments of focal elements in two ${ }^{12}$ different clusters are added up according to formulas (12)-(13).

$$
\begin{aligned}
& m_{s}^{\Omega}\left(\omega_{1}\right)=\sum_{\theta_{i} \in \omega_{1}} m^{\Theta}\left(\theta_{i}\right) \\
& m_{s}^{\Omega}\left(\omega_{2}\right)=\sum_{\theta_{j} \in \omega_{2}} m^{\Theta}\left(\theta_{j}\right)
\end{aligned}
$$

\section{Combination of the BBAs}

Based on the disagreement vector and k-means algorithm, a new adaptive bintree structure based on this flexible coarsening decomposition is obtained (see example in the next section) and the elements in FoD $\Theta$ are grouped more reasonably in each layer of the decomposition. Once the adaptive bintree structure is derived, other steps (multiplications of link weights) can be implemented which are identical to hierarchical (rigid) coarsening method presented in section II to get the final combined Bayesian BBA.

\section{Summary of the method}

The fusion method of BBAs to get a combined Bayesian BBA based on hierarchical flexible decomposition of the FoD consists of the four steps below illustrated in Fig. 2.

- Step 1 (pre-processing): At first, all input BBAs to combine are approximated to Bayesian BBAs with DSmP transform.

- Step 2 (disagreement vector): $\mathrm{D}_{1-\mathrm{S}}(\cdot)$ is calculated using $d_{B I}$ distances to estimate the degree of disagreement of BBAs $m_{1}^{\Theta}, \ldots, m_{S}^{\Theta}$ on potential decisions $\theta_{1}, \ldots, \theta_{n}$.

- Step 3 (adaptive bintree): The adaptative bintree decomposition of the FoD $\Theta$ is obtained using k-Means algorithm to get elements of subframes $\Omega_{l}$.

- Step 4 (assignments and connection weights): For each source $m_{s}^{\Theta}(\cdot)$ to combine, the mass assignment of

\footnotetext{
${ }^{10}$ For simplicity, we assume Shafer's model so that $\left|2^{\Theta}\right|=2^{n}$, otherwise the number of elements in the summation of (10) should be $\left|D^{\Theta}\right|-1$ with another normalization constant $n_{c}$.

${ }^{11}$ which is implemented in Matlab ${ }^{\mathrm{TM}}$

${ }^{12}$ because we use here the bisection decomposition.
}

each element of subframe $\Omega_{l}$ is computed by (12)-(13). The weight of links between two layers of the bintree decomposition are obtained with PCR6 rule ${ }^{13}$.

- Step 5 (fusion): The final result (combined Bayesian BBA) is computed by the product of weights of link paths from root to terminal nodes.

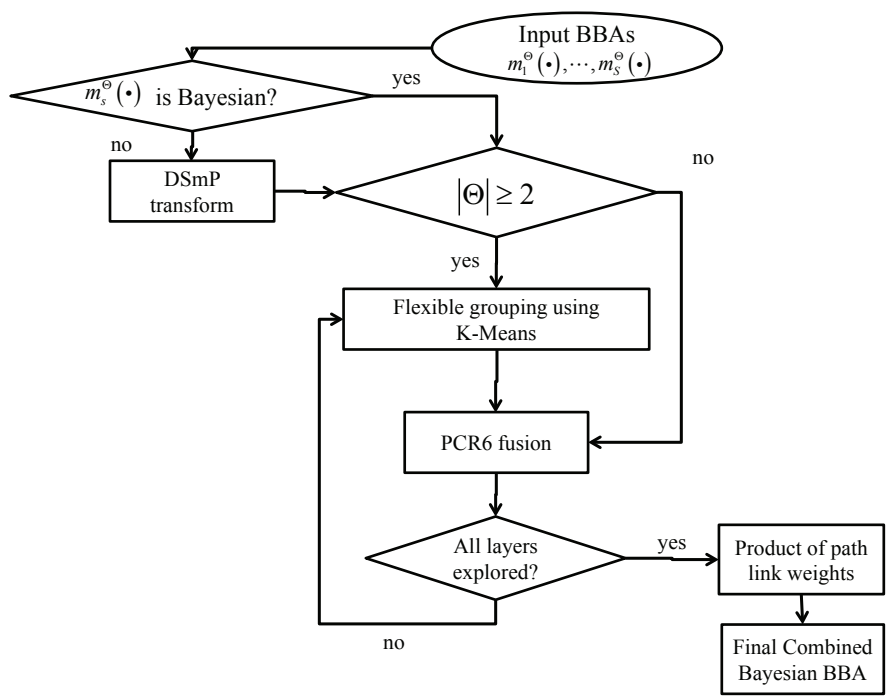

Figure 2: Hierarchical flexible decomposition of FoD for fusion.

\section{TWO SIMPLE EXAMPLES}

\section{A. Example 1 (fusion of Bayesian BBAs)}

Let us revisit example 1 presented in section II-B. It can be verified in applying formula (9) that the disagreement vector $\mathbf{D}_{1-3}$ for this example is equal to

$$
\mathbf{D}_{1-3}=[0.4085,0.2156,0.3753,0.2507,0.4086]
$$

The derivation of $D_{1-3}\left(\theta_{1}\right)$ is given below for convenience.

$$
\begin{aligned}
D_{1-3}\left(\theta_{1}\right) & =\left|d_{B I}\left(m_{1}^{\Theta}(\cdot), m_{\theta_{1}}\left(\theta_{1}\right)\right)-d_{B I}\left(m_{2}^{\Theta}(\cdot), m_{\theta_{1}}\left(\theta_{1}\right)\right)\right| \\
& +\left|d_{B I}\left(m_{2}^{\Theta}(\cdot), m_{\theta_{1}}\left(\theta_{1}\right)\right)-d_{B I}\left(m_{3}^{\Theta}(\cdot), m_{\theta_{1}}\left(\theta_{1}\right)\right)\right| \\
& +\left|d_{B I}\left(m_{1}^{\Theta}(\cdot), m_{\theta_{1}}\left(\theta_{1}\right)\right)-d_{B I}\left(m_{3}^{\Theta}(\cdot), m_{\theta_{1}}\left(\theta_{1}\right)\right)\right| \\
& =0.4085 .
\end{aligned}
$$

Based on the disagreement vector and k-means algorithm, a new adaptive bintree structure is obtained and shown in Fig. 3. Compared to Fig. 1, the elements in FoD $\Theta$ are grouped more reasonably. In vector $\mathbf{D}_{1-3}, \theta_{1}$ and $\theta_{5}$ lie in similar degree of disagreement so that they are put in the same group. Similarly for $\theta_{2}$ and $\theta_{4}$. However, element $\theta_{3}$ seems weird, which is put alone at the beginning of flexible coarsening. Once this adaptive bintree decomposition is obtained, other steps can be implemented which are identical to hierarchical coarsening method of section II to get the final combined BBA.

The flexible coarsening and fusion of BBAs is obtained from the following steps:

\footnotetext{
${ }^{13}$ general formula preferred, or applied sequentially to reduce complexity.
} 


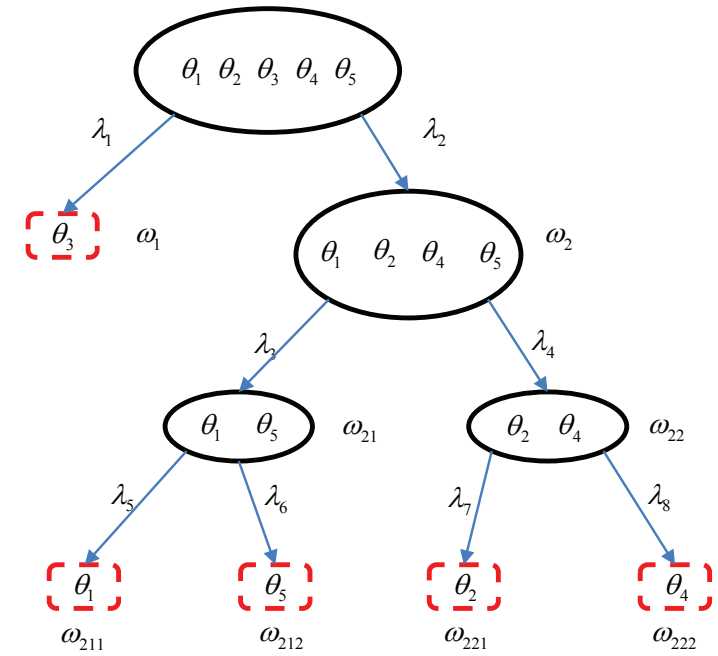

Figure 3: Example 1: Flexible bintree decomposition of FoD.

Step 1: According to Fig.3, the elements of the frames $\Omega_{l}$ are defined as follows:

- At layer $l=1: \Omega_{1}=\left\{\omega_{1} \triangleq \theta_{3}, \omega_{2} \triangleq \theta_{1} \cup \theta_{2} \cup \theta_{4} \cup \theta_{5}\right\}$

- At layer $l=2: \Omega_{2}=\left\{\omega_{21} \triangleq \theta_{1} \cup \theta_{5}, \omega_{22} \triangleq \theta_{2} \cup \theta_{4}\right\}$

- At layer $l=3$ : $\Omega_{3}=\left\{\omega_{211} \triangleq \theta_{1}, \omega_{212} \triangleq \theta_{5}, \omega_{221} \triangleq\right.$ $\left.\theta_{2}, \omega_{222} \triangleq \theta_{4}\right\}$

Step 2: The BBAs of elements of the (sub-)frames $\Omega_{l}$ are obtained as follows:

- At layer $l=1$, we use (12)-(13) and we get

\begin{tabular}{c|c|c|c} 
Focal elem. & $m_{1}^{\Omega_{1}}()$. & $m_{2}^{\Omega_{1}}()$. & $m_{3}^{\Omega_{1}}()$. \\
\hline$\omega_{1} \triangleq \theta_{3}$ & 0.3 & 0.1 & 0.5 \\
$\omega_{2} \triangleq \theta_{1} \cup \theta_{2} \cup \theta_{4} \cup \theta_{5}$ & 0.7 & 0.9 & 0.5
\end{tabular}

- At layer $l=2$ : We use again the proportional redistribution method which gives us:

\begin{tabular}{c|c|c|c} 
Focal elem. & $m_{1}^{\Omega_{2}}()$. & $m_{2}^{\Omega_{2}}()$. & $m_{3}^{\Omega_{2}}()$. \\
\hline$\omega_{21} \triangleq \theta_{1} \cup \theta_{5}$ & $\frac{3}{7}$ & $\frac{4}{9}$ & $\frac{1}{5}$ \\
$\omega_{22} \triangleq \theta_{2} \cup \theta_{4}$ & $\frac{4}{7}$ & $\frac{5}{9}$ & $\frac{4}{5}$
\end{tabular}

- At layer $l=3$ : We work with the two subframes $\Omega_{31} \triangleq$ $\left\{\omega_{211}, \omega_{212}\right\}$ and $\Omega_{32} \triangleq\left\{\omega_{221}, \omega_{222}\right\}$ of $\Omega_{3}$ with the BBAs

\begin{tabular}{c|c|c|c} 
Focal elem. & $m_{1}^{\Omega_{31}}()$. & $m_{2}^{\Omega_{31}}()$. & $m_{3}^{\Omega_{31}}()$. \\
\hline$\omega_{211} \triangleq \theta_{1}$ & $\frac{1}{2}$ & $\frac{1}{2}$ & $\frac{1}{2}$ \\
$\omega_{212} \triangleq \theta_{5}$ & $\frac{1}{2}$ & $\frac{1}{2}$ & $\frac{1}{2}$ \\
Focal elem. & $m_{1}^{\Omega_{32}}()$. & $m_{2}^{\Omega_{32}}()$. & $m_{3}^{\Omega_{32}}()$. \\
\hline$\omega_{221} \triangleq \theta_{2}$ & $\frac{2}{5}$ & 0 & $\frac{1}{5}$ \\
$\omega_{222} \triangleq \theta_{4}$ & $\frac{3}{5}$ & 1 & $\frac{4}{5}$
\end{tabular}

Step 3: The connection weights $\lambda_{i}$ are computed from the assignments of coarsening elements. Hence, we obtain the following connecting weights in the bintree:

- At layer $l=1$ :

$$
\lambda_{1}=0.2226 ; \quad \lambda_{2}=0.7774 .
$$

- At layer $l=2$ :

$$
\lambda_{3}=0.2200 ; \quad \lambda_{4}=0.7800 .
$$

- At layer $l=3$ :

$$
\lambda_{5}=0.5 ; \quad \lambda_{6}=0.5 ; \lambda_{7}=0.0669 ; \quad \lambda_{8}=0.9331 .
$$

Step 4: We finally get the following resulting combined and normalized Bayesian BBA

$$
m^{\Theta}(\cdot)=\{0.0855,0.0406,0.2226,0.5658,0.0855\} .
$$

\section{B. Example 2 (with non-Bayesian BBAs)}

Example 1bis: Let's consider $\Theta=\left\{\theta_{1}, \theta_{2}, \theta_{3}, \theta_{4}, \theta_{5}\right\}$, and the following BBAs given by

\begin{tabular}{c|ccc} 
Focal elem. & $m_{1}^{\Theta}()$. & $m_{2}^{\Theta}()$. & $m_{3}^{\Theta}()$. \\
\hline$\theta_{1}$ & 0.1 & 0.4 & 0 \\
$\theta_{2}$ & 0.2 & 0 & 0 \\
$\theta_{3}$ & 0.3 & 0.05 & 0 \\
$\theta_{4}$ & 0.03 & 0.05 & 0 \\
$\theta_{5}$ & 0.1 & 0.04 & 0 \\
$\theta_{1} \cup \theta_{2}$ & 0.1 & 0.04 & 0 \\
$\theta_{2} \cup \theta_{3} \cup \theta_{5}$ & 0 & 0.02 & 0.1 \\
$\theta_{3} \cup \theta_{4}$ & 0.02 & 0.1 & 0.2 \\
$\theta_{1} \cup \theta_{5}$ & 0.1 & 0.3 & 0.2 \\
$\Theta$ & 0.05 & 0 & 0.5
\end{tabular}

Step 1 (Pre-Processing): All these three BBAs are transformed into Bayesian BBAs with DSmP transform and the generated BBAs are illustrated as

\begin{tabular}{c|ccc} 
Focal elem. & $m_{1}^{\Theta}()$. & $m_{2}^{\Theta}()$. & $m_{3}^{\Theta}()$. \\
\hline$\theta_{1}$ & 0.1908 & 0.7127 & 0.2000 \\
$\theta_{2}$ & 0.2804 & 0 & 0.1334 \\
$\theta_{3}$ & 0.3387 & 0.1111 & 0.2333 \\
$\theta_{4}$ & 0.0339 & 0.1 & 0.2000 \\
$\theta_{5}$ & 0.1562 & 0.0761 & 0.2333
\end{tabular}

It can be verified in applying formula (9) that the disagreement vector $\mathbf{D}_{1-3}$ for this example is equal to

$$
\mathbf{D}_{1-3}=[0.5385,0.3632,0.3453,0.2305,0.2827]
$$

Step 2: According to the clustering algorithm, the elements of the frames $\Omega_{l}$ are defined as follows:

- At layer $l=1: \Omega_{1}=\left\{\omega_{1} \triangleq \theta_{1}, \omega_{2} \triangleq \theta_{2} \cup \theta_{3} \cup \theta_{4} \cup \theta_{5}\right\}$

- At layer $l=2: \Omega_{2}=\left\{\omega_{21} \triangleq \theta_{2} \cup \theta_{3}, \omega_{22} \triangleq \theta_{4} \cup \theta_{5}\right\}$

- At layer $l=3: \Omega_{3}=\left\{\omega_{211} \triangleq \theta_{2}, \omega_{212} \triangleq \theta_{3}, \omega_{221} \triangleq\right.$ $\left.\theta_{4}, \omega_{222} \triangleq \theta_{5}\right\}$

Step 3: The BBAs of elements of the (sub-)frames $\Omega_{l}$ are obtained as follows:

- At layer $l=1$, we use (12)-(13) and we get

\begin{tabular}{c|c|c|c} 
Focal elem. & $m_{1}^{\Omega_{1}}()$. & $m_{2}^{\Omega_{1}}()$. & $m_{3}^{\Omega_{1}}()$. \\
\hline$\omega_{1} \triangleq \theta_{1}$ & 0.1908 & 0.7127 & 0.2000 \\
$\omega_{2} \triangleq \theta_{2} \cup \theta_{3} \cup \theta_{4} \cup \theta_{5}$ & 0.8092 & 0.2873 & 0.8000
\end{tabular}

- At layer $l=2$ : We use again the proportional redistribution method which gives us:

\begin{tabular}{c|c|c|c} 
Focal elem. & $m_{1}^{\Omega_{2}}()$. & $m_{2}^{\Omega_{2}}()$. & $m_{3}^{\Omega_{2}}()$. \\
\hline$\omega_{21} \triangleq \theta_{2} \cup \theta_{3}$ & 0.7651 & 0.3867 & 0.4584 \\
$\omega_{22} \triangleq \theta_{4} \cup \theta_{5}$ & 0.2349 & 0.6133 & 0.5416
\end{tabular}


- At layer $l=3$ : We work with the two subframes $\Omega_{31} \triangleq$ $\left\{\omega_{211}, \omega_{212}\right\}$ and $\Omega_{32} \triangleq\left\{\omega_{221}, \omega_{222}\right\}$ of $\Omega_{3}$ with the BBAs:

\begin{tabular}{c|c|c|c} 
Focal elem. & $m_{1}^{\Omega_{31}}()$. & $m_{2}^{\Omega_{31}}()$. & $m_{3}^{\Omega_{31}}()$. \\
\hline$\omega_{211} \triangleq \theta_{2}$ & 0.4529 & 0 & 0.3638 \\
$\omega_{212} \triangleq \theta_{3}$ & 0.5471 & 1 & 0.6362 \\
Focal elem. & $m_{1}^{\Omega_{32}}()$. & $m_{2}^{\Omega_{32}}()$. & $m_{3}^{\Omega_{32}}()$. \\
\hline$\omega_{221} \triangleq \theta_{4}$ & 0.1783 & 0.5679 & 0.4616 \\
$\omega_{222} \triangleq \theta_{5}$ & 0.8217 & 0.4321 & 0.5384
\end{tabular}

Step 4: The connection weights $\lambda_{i}$ are computed from the assignments of coarsening elements. Hence, we obtain the following connecting weights in the bintree:

- At layer $l=1$ :

$$
\lambda_{1}=0.2345 ; \quad \lambda_{2}=0.7655 .
$$

- At layer $l=2$ :

$$
\lambda_{3}=0.5533 ; \quad \lambda_{4}=0.4467 .
$$

- At layer $l=3$ :

$$
\begin{array}{ll}
\lambda_{5}=0.1606 ; & \lambda_{6}=0.8394 ; \\
\lambda_{7}=0.3349 ; & \lambda_{8}=0.6651 .
\end{array}
$$

Step 5: We finally get the following resulting combined and normalized Bayesian BBA

$$
m^{\Theta}(\cdot)=\{0.2345,0.0681,0.3555,0.1145,0.2274\} .
$$

\section{SIMULATION RESUlTS AND PERFORMANCES}

\section{A. Flexible Grouping of Singletons}

1) Similarity: ${ }^{14}$ Assuming that $\Theta=\left\{\theta_{1}, \theta_{2}, \theta_{3}, \theta_{4}, \theta_{5}, \theta_{6}\right.$, $\left.\theta_{7}, \theta_{8}, \theta_{9}, \theta_{10}, \theta_{11}, \theta_{12}, \theta_{13}, \theta_{14}, \theta_{15}\right\}$ and first, we randomly generate 2 BBAs, denoted as $m_{1}^{\Theta}(\cdot)$ and $m_{2}^{\Theta}(\cdot)$, which can be seen in Table I.

Table I: BBAs for Two Sources $m_{1}^{\Theta}(\cdot)$ and $m_{2}^{\Theta}(\cdot)$

\begin{tabular}{cccccc}
\hline & $\theta_{1}$ & $\theta_{2}$ & $\theta_{3}$ & $\theta_{4}$ & $\theta_{5}$ \\
\hline$m_{1}^{\Theta}(\cdot)$ & 0.1331 & 0.0766 & 0.0175 & 0.0448 & 0.0229 \\
$m_{2}^{\Theta}(\cdot)$ & 0.1020 & 0.0497 & 0.1094 & 0.0612 & 0.0612 \\
\hline
\end{tabular}

\begin{tabular}{cccccc}
\hline & $\theta_{6}$ & $\theta_{7}$ & $\theta_{8}$ & $\theta_{9}$ & $\theta_{10}$ \\
\hline$m_{1}^{\Theta}(\cdot)$ & 0.1142 & 0.0023 & 0.2254 & 0.1583 & $3.4959 \mathrm{e}-04$ \\
$m_{2}^{\Theta}(\cdot)$ & 0.0069 & 0.0070 & 0.0128 & 0.0833 & 0.0338 \\
\hline
\end{tabular}

\begin{tabular}{cccccc}
\hline & $\theta_{11}$ & $\theta_{12}$ & $\theta_{13}$ & $\theta_{14}$ & $\theta_{15}$ \\
\hline$m_{\ominus}^{\ominus}(\cdot)$ & 0.0075 & 0.0514 & 0.1121 & 0.0314 & 0.0021 \\
$m_{2}^{\ominus}(\cdot)$ & 0.1180 & 0.1202 & 0.1351 & 0.0686 & 0.0309 \\
\hline
\end{tabular}

In order to fully verify the similarity between hierarchical flexible coarsening method and PCR6 in DSmT, a new strict

\footnotetext{
${ }^{14}$ Similarity represents the approximate degree between fusion results using flexible coarsening and PCR6.
}

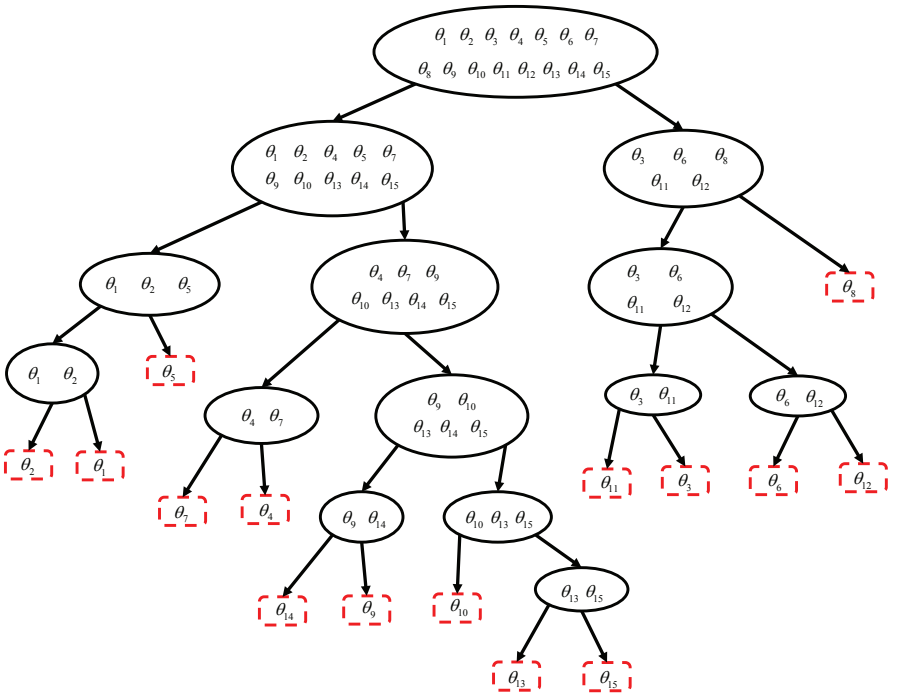

Figure 4: Structure of Hierarchical Flexible Coarsening.

distance metric between two BBAs, denoted $d_{B I}^{E}$, was recently proposed in [20], [16] and it will be used in this paper.

In this paper, we regard $d_{B I}^{E}$ as one criteria for evaluating the degree of similarity between the fusion results obtained from flexible coarsening and PCR6.

Based on (8) and (10), the disagreement vector $\mathbf{D}(\cdot)$ is obtained:

$$
\begin{aligned}
& \mathbf{D}(\cdot)=(0.0032,0.0020,0.0290,0.0092,0.0147,0.0228, \\
& \quad 0.0059,0.0537,0.0154,0.0131,0.0338,0.0235, \\
& \\
& 0.0118,0.0145,0.0120) .
\end{aligned}
$$

Thus, bintree structure of hierarchical flexible coarsening is illustrated in Fig. 4 and the similarity between fusion results of hierarchical flexible coarsening and PCR6 is 0.9783. And the similarity between hierarchical coarsening method and PCR6 is 0.9120 . In particular, terminal nodes (the red small box in Fig. 4) of flexible grouping are not in accordance with the original order $\theta_{1}, \theta_{2}, \cdots, \theta_{15}$. This is quite different compared to original hierarchical coarsening method.

From the point of view of statistics, 100 BBAs are randomly generated to be fused with three methods: hierarchical flexible coarsening, hierarchical coarsening and also PCR6. Comparisons are made in Fig. 5, which show the superiority of our new approach proposed in this paper (Average value of new method is $\mathbf{9 7 \%}$ and the old method is $\mathbf{9 3 . 5 \%}$ ).

\section{B. Flexible Grouping of Conflicting Focal Elements}

Assuming that there are five sources of evidence $m_{1}^{\Theta}(\cdot), m_{2}^{\Theta}(\cdot), m_{3}^{\Theta}(\cdot), m_{4}^{\Theta}(\cdot), m_{5}^{\Theta}(\cdot)$, and the restricted hypepower set $D^{\Theta}=\left\{\theta_{1}, \theta_{2}, \theta_{3}, \theta_{4}, \theta_{5}, \theta_{6}, \theta_{7}, \theta_{8}, \theta_{9}, \theta_{10}, \theta_{1} \cap\right.$ $\left.\theta_{2}, \theta_{5} \cap \theta_{6} \cap \theta_{7}, \theta_{1} \cap \theta_{5} \cap \theta_{9} \cap \theta_{10}\right\}$. And then we randomly generate $1000 \mathrm{BBAs}$ for each source to calculate the similarity using (10). From Fig. 6, we can find that hierarchical flexible coarsening method can also maintain high degree of similarity which performs better than hierarchical coarsening. 


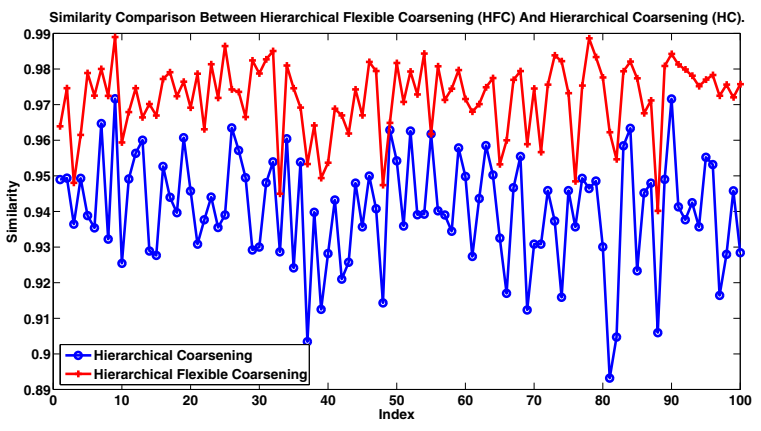

Figure 5: Comparisons Between HFC and HC (Only Singletons).

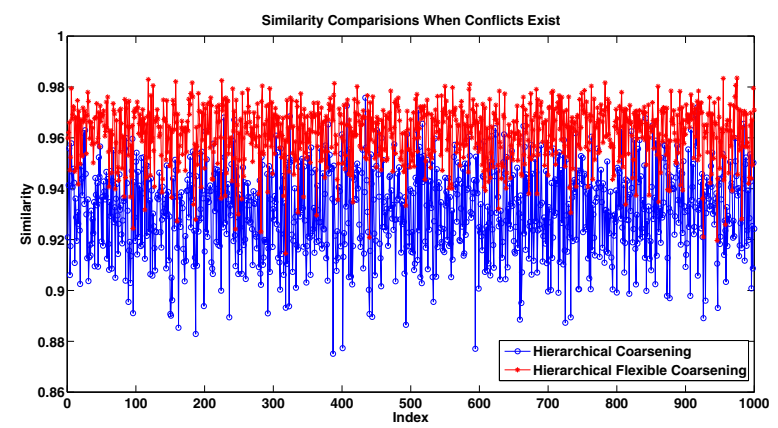

Figure 6: Comparisons Between HFC and HC (Singletons and Conflicting Focal Elements).

\section{Flexible Grouping of Uncertain and Hybrid Focal Elements}

We can also deal with uncertain and hybrid focal elements. Assuming that there are also five sources of evidence $m_{1}^{\Theta}(\cdot), m_{2}^{\Theta}(\cdot), m_{3}^{\Theta}(\cdot), m_{4}^{\Theta}(\cdot), m_{5}^{\Theta}(\cdot)$ and $D_{1}^{\Theta}=$ $\left\{\theta_{1}, \theta_{2}, \theta_{3}, \theta_{4}, \theta_{5}, \theta_{6}, \theta_{7}, \theta_{8}, \theta_{9}, \theta_{10}, \theta_{1} \cup \theta_{2}, \theta_{5} \cup \theta_{6} \cup \theta_{7}, \theta_{1} \cup\right.$ $\left.\theta_{5} \cup \theta_{9} \cup \theta_{10}\right\} ; D_{2}^{\Theta}=\left\{\theta_{1}, \theta_{2}, \theta_{3}, \theta_{4}, \theta_{5}, \theta_{6}, \theta_{7}, \theta_{8}, \theta_{9}, \theta_{10}, \theta_{2} \cap\right.$ $\left.\theta_{4} \cup \theta_{6}, \theta_{1} \cup \theta_{3} \cap \theta_{5} \cup \theta_{7} \cap \theta_{9}\right\}^{15}$. And then we respectively and randomly generate 1000 BBAs for these two cases $D_{1}^{\Theta}$ and $D_{2}^{\Theta}$. Finally, we calculate the average similarity degree of HFC and HC with PCR6 in Table II, which illustrates HFC performs better than old method. However, there exist the extra time cost of HFC compared to HC due to the clustering steps in coarsening process.

Table II: Similarity Comparisons

\begin{tabular}{ccc}
\hline & Hierarchical Flexible Coarsening & Hierarchical Coarsening \\
\hline$D_{1}^{\Theta}$ & $98 \%$ & $91 \%$ \\
$D_{2}^{\Theta}$ & $97 \%$ & $93 \%$ \\
\hline
\end{tabular}

\section{CONCLUSION AND PERSPECTIVES}

A novel hierarchical flexible approximate method in DSmT is proposed here. Compared to original hierarchical coarsen-

\footnotetext{
${ }^{15}$ In this case, $D_{1}^{\Theta}$ represents uncertain focal elements and $D_{2}^{\Theta}$ represents hybrid focal elements.
}

ing, flexible strategy guarantees higher similarity with PCR6 rules in fusion process. Besides, whether focal elements in hyper power set are singletons, conflicting focal elements, uncertain or even hybrid focal elements, the new method works well. In the future work, we will focus on the general framework of hierarchical coarsening, which could generate final non-Bayesian BBAs in order to avoid loss of information. Furthermore, other advantages or disadvantages of our proposed methods such as computational efficiency and time consumption need to be further investigated.

\section{ACKNOWLEDGMENT}

This work was supported by NNSF of China (No.61175091), Qing Lan Project of Jiangsu Province, Aeronautical Science Foundation of China (20140169002), and Six Major Top-talent Plan of Jiangsu Province.

\section{REFERENCES}

[1] G. Shafer, A mathematical theory of evidence, Princeton Univ. Press, 1976.

[2] A. Dempster, Upper and lower probabilities induced by a multivalued mapping, in Annals of Mathematical Statistics, Vol. 38, pp. 325-339, 1967.

[3] P. Smets, Practical uses of belief functions, in K.B. Laskey and H. Prade Editors, 15th Conf. on Uncertainty in Artificial Intelligence, pp. 612-621, Stockholm, Sweden, 1999.

[4] J. Dezert, Foundations for a new theory of plausible and paradoxical reasoning, in Information \& Security: An Int. Journal, Vol. 9, 2002.

[5] F. Smarandache, J. Dezert (Editors), Advances and applications of DSmT for information fusion, American Research Press, Rehoboth, NM, U.S.A., Vol. 1-4, 2004-2015. Available at webpage 2 of [6].

[6] http://www.onera.fr/staff/jean-dezert

[7] R. Kennes, Computational aspects of the Möbius transform of graphs, in IEEE Trans. on SMC, Vol. 22, pp. 201-223, 1992.

[8] G. Shafer, R. Logan, Implementing Dempster's rule for hierarchical evidence, in Artificial Intelligence, Vol. 33, pp. 271-298, 1987.

[9] Y. Yang, Y.L. Liu, Iterative approximation of basic belief assignment based on distance of evidence, in Plos One, Vol. 11, No. 2, 2016.

[10] T. Denœux, Inter and outer approximation of belief structures using a hierarchical clustering approach, in Int. J. of Uncertainty, Fuzziness and Knowledge-Based System, Vol. 9, No 4, pp. 437-460, 2001.

[11] Y. Yang, D. Han, C. Han, F. Cao, A novel approximation of basic probability assignment based on rank-level fusion, in Chinese Journal of Aeronautics, Vol. 26, No. 4, pp. 993-999, 2013.

[12] D. Han, Y. Yang, J. Dezert, Two novel methods of BBA approximation based on focal element redundancy, in Proc. of Fusion 2015, Washington, D.C., USA, July 2015.

[13] P. Smets, Analyzing the combination of conflicting belief functions, in Information Fusion, Vol. 8, pp. 387-412, 2006.

[14] P. Smets, Decision making in the TBM: the necessity of the pignistic transformation, in Int. J. of Approx. reasoning, Vol. 38, 2005.

[15] J. Dezert, F. Smarandache, A new probabilistic transformation of belief mass assignment, in Proc. of Fusion 2008.

[16] J. Dezert, D. Han, J.-M. Tacnet, S. Carladous, Y. Yang, Decision-making with belief interval distance, in Proc. of Belief 2016 Int. Conf., Prague, CZ, 2016.

[17] X.D. Li, J. Dezert, X.H. Huang, Z.D. Meng, X.J. Wu, A fast approximate reasoning method in hierarchical DSmT (A), in Acta Electronica Sinica, Vol. 38, No. 11, pp. 2567-2572, 2010.

[18] X.D. Li, W.D. Yang, X.J. W, J. Dezert, A fast approximate reasoning method in hierarchical DSmT (B), in Acta Electronica Sinica, Vol. 39, No. 3A, pp. 32-36, 2011.

[19] X.D. Li, W.D. Yang, J. Dezert, A fast approximate reasoning method in hierarchical DSmT (C), in J. Huazhong Univ. of Sci. and Tech. (Natural Science Edition), Vol. 39, pp. 151-156, 2011.

[20] D. Han, J., Dezert, Y., Yang, New distance measures of evidence based on belief intervals, in Proc. of Belief 2014, Oxford, UK, 2014. 